\title{
TWO RESULTS ON C-CONGRUENCE NUMERICAL RADII
}

\author{
CHE-MAN CHENG
}

\begin{abstract}
Let $M_{n}$ denote the set of $n \times n$ complex matrices. For $A$ and $C$ in $M_{n}$, define the $C$-congruence numerical radius of $A$ by

$$
\rho_{C}(A)=\max \left\{\left|\operatorname{tr}\left(C U A U^{t}\right)\right|: U \text { is unitary }\right\} .
$$

First, we show that $\rho c$ is a norm on $M_{n}$ if and only if $C$ is neither symmetric nor skew-symmetric. Secondly, we use $\rho c$ to characterize two matrices $A$ and $B$ in $M_{n}$ to be unitarily congruent (i.e. $A=U B U^{t}$ for some unitary $U$ ).
\end{abstract}

\section{Introduction.}

Let $M_{n}$ denote the set of all $n \times n$ complex matrices and $\mathcal{U}_{n}$ the subset consisting of all $n \times n$ unitary matrices. Denote by $\operatorname{tr} A, A^{t}$ and $A^{*}$ the trace, transpose and conjugate transpose of $A\left(\in M_{n}\right)$ respectively. For $A$ and $C$ in $M_{n}$, the C-numerical range of $A$ and the $\mathrm{C}$-numerical radius of $A$ defined by

$$
W_{C}(A)=\left\{\operatorname{tr}\left(C U A U^{*}\right): U \in \mathcal{U}_{n}\right\}
$$

and

$$
r_{C}(A)=\max \left\{|z|: z \in W_{C}(A)\right\}
$$

respectively have been studied extensively. In particular, Goldberg and Straus [2] showed that $r_{C}$ is a norm on $M_{n}$ if and only if $C$ is nonscalar and $\operatorname{tr} C \neq 0$ (for other proofs, see [6] and [8]). Li and Tsing [5] showed that $A=\alpha U B U^{*}$ with $\alpha \in \mathcal{C}$ and $|\alpha|=1$ if and only if $r_{A^{*}}(A)=r_{A^{*}}(B)$ and $r_{B^{*}}(A)=r_{B^{*}}(B)$.

Parallel to the $\mathrm{C}$-numerical range, Thompson [10] introduced the $\mathrm{C}$-congruence numerical range of $A$ defined by

$$
R_{C}(A)=\left\{\operatorname{tr}\left(C U A U^{t}\right): U \in \mathcal{U}_{n}\right\}
$$

Various results have also been obtained, see [1] and [9].

Received April 11, 1989.

The author wishes to express his thanks to Dr. Yik-Hoi Au-Yeung for his valuable advice and encouragement. 
It is natural to define the $\mathrm{C}$-congruence numerical radius of $A$ by

$$
\rho_{C}(A)=\max \left\{|z|: z \in R_{C}(A)\right\} .
$$

By taking $C$ to be any symmetric matrix (i.e. $C=C^{t}$ ) and $A$ to be any skew-symmetric matrix (i.e. $A=-A^{t}$ ), or vice versa, we can easily check that $\rho_{C}(A)=0$. So, $\rho_{C}$ is not a norm of $M_{n}$ if $C$ is symmetric or skew symmetric. We shall prove, for other cases of $C, \rho_{C}$ is a norm on $M_{n}$.

Two matrices $A$ and $B$ are said to be unitarily congruent (resp. unitarily similar) if $A=U B U^{t}$ (resp. $A=U B U^{*}$ ) for some $U \in \mathcal{U}_{n}$. Hong and Horn [3] gave a characterization for two matrices to be unitarily congruent in terms of unitary similarities. As an application of $\rho_{C}$, we shall prove that $A$ and $B$ are unitarily congruent if and only if $\rho_{A^{*}}(A)=\rho_{A^{*}}(B)$ and $\rho_{B^{*}}(A)=\rho_{B^{*}}(B)$.

In what follows, we shall assume $n>1$ so as to avoid trivial modifications.

2. Two results on $\mathrm{C}$-congruence numerical radii.

Since the relation $\langle A, B\rangle=\operatorname{tr}\left(A B^{*}\right)$ is an inner product on $M_{n}$, the following lemma is obvious.

Lemma 1. Let $C \in M_{n}$. Then the following two statements are equivalent:

(i) For any $A \in M_{n}$, if $\rho_{C}(A)=0$, then $A=0$;

(ii) $\operatorname{span}\left\{U^{t} C U: U \in \mathcal{U}_{n}\right\}=M_{n}$, where $\operatorname{span}\left\{U^{t} C U: U \in \mathcal{U}_{n}\right\}$ is the linear subspace of $M_{n}$ spanned by the set $\left\{U^{t} C U: U \in \mathcal{U}_{n}\right\}$.

Let $\mathcal{S}_{n}$ (resp. $\mathcal{K}_{n}$ ) denote the set of all $n \times n$ symmetric (resp. skew-symmetric) matrices. Let $\operatorname{diag}\left(s_{1}, \cdots, s_{n}\right)$ denote the diagonal matrix with the $i^{\text {th }}$ diagonal entry being $s_{i}$. The direct sum of two square matrices $A$ and $B$ is written as $A \oplus B$. The following two lemmas are due to Takagi [7] and Youla[11] respectively.

Lemma 2. Let $C \in \mathcal{S}_{n}$. Then there exists $U \in \mathcal{U}_{n}$ such that $U^{t} C U=$ $\operatorname{diag}\left(s_{1}, \cdots, s_{n}\right)$, where $s_{i}, i=1, \cdots, n$ are non-negative real numbers and $s_{i} \geq s_{i+1}, i=$ $1, \cdots, n-1$.

Lemma. 3. Let $C \in \mathcal{K}_{n}$. Then there exists $U \in \mathcal{U}_{n}$ such that

$$
U^{i} C U=\left(\begin{array}{cc}
0 & s_{1} \\
-s_{1} & 0
\end{array}\right) \oplus \cdots \oplus\left(\begin{array}{cc}
0 & s_{m} \\
-s_{m} & 0
\end{array}\right) \oplus[0] \oplus \cdots \oplus[0]
$$

where $s_{i}, i=1, \cdots, m$ are positive real numbers and $s_{i} \geq s_{i+1}, i=1, \cdot, m-1$.

Let $E_{i j}$ denote the matrix in $M_{n}$ with 1 at its $(i, j)$ entry and zero elsewhere. If $\sigma$ is a permutation of $\{1, \cdots, n\}$, then $P(\sigma)$ denotes the corresponding permutation matrix (i.e. $\left.P(\sigma)=\left(\delta_{i, \sigma(i)}\right)\right)$. We also use the standard notation $\left(i_{1}, \cdots, i_{r}\right)$ to denote the 
permutation (in fact, a cycle) $\sigma$ for which $\sigma\left(i_{l}\right)=i_{l+1}$ for $l=1, \cdots, r-1, \sigma\left(i_{r}\right)=i_{1}$ and $\sigma(i)=i$ otherwise. As usual, $\sigma_{1} \sigma_{2}$ denotes the composition of $\sigma_{1}$ and $\sigma_{2}$.

Tam[8] showed that if $A$ is nonscalar and $\operatorname{tr} A \neq 0$, then $\operatorname{span}\left\{U A U^{*}: U \in \mathcal{U}_{n}\right\}=$ $M_{n}$. The following Theorem gives a parallel result.

Theorem 1. Let $C(\neq 0) \in M_{n}$. Then

$$
\operatorname{span}\left\{U^{t} C U: U \in \mathcal{U}_{n}\right\}= \begin{cases}\mathcal{S}_{n} & \text { if } C \in \mathcal{S}_{n} \\ \mathcal{K}_{n} & \text { if } C \in \mathcal{K}_{n} \\ M_{n} & \text { otherwise. }\end{cases}
$$

Proof. Case 1. $C \in \mathcal{S}_{n}$, then the inclusion $\operatorname{span}\left\{U^{t} C U: U \in \mathcal{U}_{n}\right\} \subset \mathcal{S}_{n}$ is trival. Since span $\left\{U^{t} C U: U \in \mathcal{U}_{n}\right\}$ is invariant under unitary congruence of $C$, by Lemma 2, we may assume $C=\operatorname{diag}\left(s_{1}, \cdots, s_{n}\right)$.

Firstly, suppose that $C=E_{11}$. Let

$$
V=\left(\frac{1}{\sqrt{2}}\left(\begin{array}{cc}
1 & 1 \\
1 & -1
\end{array}\right)\right) \oplus I_{n-2} \in \mathcal{U}_{n}
$$

where $I_{n-2}$ is the $(n-2) \times(n-2)$ identity matrix. Then

$$
V^{t} C V=\left(\frac{1}{2}\left(\begin{array}{ll}
1 & 1 \\
1 & 1
\end{array}\right)\right) \oplus O_{n-2}
$$

where $O_{n-2}$ is the $(n-2) \times(n-2)$ zero matrix. Direct computations yield

$$
\begin{gathered}
P^{t}((1 j)) C P((1 j))=E_{j j}, j=1, \cdots, n ; \\
P^{t}((1 r)(2 l))\left(V^{t} C V\right) P((1 r)(2 l))=\frac{1}{2}\left(E_{r r}+E_{r l}+E_{l r}+E_{l l}\right): 1 \leq r<l \leq n .
\end{gathered}
$$

As the multiplication of permutation matrices in this way preserves unitary congruence, we have shown that $\left.\left\{E_{j j}: j=1, \cdots, n\right\} \cup \frac{1}{2}\left(E_{r r}+E_{r l}+E_{l r}+E_{l l}\right): 1 \leq r<l \leq n\right\} \subset$ $\left\{U^{t} C U: U \in \mathcal{U}_{n}\right\}$. Since they also form a basis of $\mathcal{S}_{n}$, we are done if $C=E_{11}$.

For the general case $C=\operatorname{diag}\left(s_{1}, \cdots, s_{n}\right)$ with $s_{1} \neq 0($ else $C=0)$ let $W=$ $\operatorname{diag}(1, i, \cdots, i) \in \mathcal{U}_{n}$, where $i^{2}=-1$. Then

$$
\frac{1}{2 s_{1}}\left(W^{t} C W+C\right)=E_{11} .
$$

Hence, we have

$$
\operatorname{span}\left\{U^{t} C U: U \in \mathcal{U}_{n}\right\} \supseteq \operatorname{span}\left\{U^{t} E_{11} U: U \in \mathcal{U}_{n}\right\}=\mathcal{S}_{n}
$$

and so Case 1 is settled. 
Case 2. $C \in \mathbb{K}_{n}$. The proof is similar to case 1 and we give only a sketch. We apply Lemma 3 , if $C=\left(\begin{array}{cc}0 & 1 \\ -1 & 0\end{array}\right) \oplus O_{n-2}$, then

$$
\left\{P^{t}((1 r)(2 l)) C P((1 r)(2 l)): 1 \leq r<l \leq n\right\}=\left\{\left(E_{r l}-E_{l r}\right): 1 \leq r<l \leq n\right\}
$$

and is a basis of $\mathcal{K}_{n}$. For the general case, we let

$$
W= \begin{cases}I_{2} \oplus\left(\begin{array}{ll}
0 & 1 \\
1 & 0
\end{array}\right) \oplus \cdots \oplus\left(\begin{array}{ll}
0 & 1 \\
1 & 0
\end{array}\right) & \text { if } \mathrm{n} \text { is even } \\
I_{2} \oplus\left(\begin{array}{ll}
0 & 1 \\
1 & 0
\end{array}\right) \oplus \cdots \oplus\left(\begin{array}{ll}
0 & 1 \\
1 & 0
\end{array}\right) \oplus[1] & \text { if } \mathrm{n} \text { is odd }\end{cases}
$$

Then $\frac{1}{2 s_{1}}\left(W^{t} C W+C\right)=\left(\begin{array}{cc}0 & 1 \\ -1 & 0\end{array}\right) \oplus O_{n-2}$. Hence our result follows.

Case 3. $C$ is neither symmetric nor skew-symmetric.

Claim: there exist $\lambda_{i} \in \mathcal{C}$ and $U_{i} \in \mathcal{U}_{n}, i=1, \cdots, l$ such that $\Sigma_{i=1}^{l} \lambda_{i} U_{i}^{t} C U_{i}=C^{t}$.

Proof. Since $C+C^{t}$ is symmetric and non-zero, by case 1 , we can find $V_{1}, \cdots, V_{m} \in$ $\mathcal{U}_{n}$, where $m=1+\cdots+n=\operatorname{dim} \mathcal{S}_{n}$, such that

$$
\left\{V_{i}^{t}\left[\frac{1}{2}\left(C+C^{t}\right)\right] V_{i}: i=1, \cdots, m\right\}
$$

is a basis of $\mathcal{S}_{n}$. Since $\operatorname{dim} \mathcal{S}_{n}>\operatorname{dim} \mathcal{K}_{n}$,

$$
\left\{V_{i}^{t}\left[\frac{1}{2}\left(C-C^{t}\right)\right] V_{i}: i=1, \cdots, m\right\}
$$

must be linearly dependent. Let $\beta_{i} \in \mathcal{C}, i=1, \cdots, m$ be such that not all of them are zero and

$$
\sum_{i=1}^{m} \beta_{i} V_{i}^{t}\left[\frac{1}{2}\left(C-C^{t}\right)\right] V_{i}=0 .
$$

Then, as $C=\frac{1}{2}\left(C+C^{t}\right)+\frac{1}{2}\left(C-C^{t}\right)$,

$$
\sum_{i=1}^{m} \beta_{i} V_{i}^{t} C V_{i}=\sum_{i=1}^{m} \beta_{i} V_{i}^{t}\left[\frac{1}{2}\left(C+C^{t}\right)\right] V_{i}
$$

is symmetric and nonzero. By case 1 again, there exists $\alpha_{j} \in \mathcal{C}$ and $U_{j} \in \mathcal{U}_{n}, j=1, \cdots, r$ such that

$$
\sum_{j=1}^{r} \alpha_{j} U_{j}^{t}\left(\sum_{i=1}^{m} \beta_{i} V_{i}^{t} C V_{i}\right) U_{j}=C+C^{t}
$$

Hence the claim is valid. 
Now, for any $A \in M_{n}, A=\frac{1}{2}\left(A+A^{t}\right)+\frac{1}{2}\left(A-A^{t}\right)$.

With $\sum_{i=1}^{l} \lambda_{i} U_{i}^{t} C U_{i}=C^{t}$, by Case 1 and Case 2 respectively, we conclude that

$$
\frac{1}{2}\left(A+A^{t}\right), \frac{1}{2}\left(A-A^{t}\right) \in \operatorname{span}\left\{U^{t} C U: U \in \mathcal{U}_{n}\right\} .
$$

Hence $A \in \operatorname{span}\left\{U^{t} C U: U \in \mathcal{U}_{n}\right\}$ and the proof is completed.

By Lemma 1 and Theorem 1, we readily have:

Theorem 2. For $n>1, \rho_{C}$ is a norm on $M_{n}$ if and only if $C$ is neither symmetric nor skew-symmetric.

From Theorem 1 and results similar to Lemma 1 , we see that if $C(\not \neq)$ is symmetric (resp. skew-symmetric), $\rho_{C}$ is a norm on $\mathcal{S}_{n}$ (resp. $\mathcal{K}_{n}$ ).

The idea of the proof of the following theorem was given by Li and Tsing [5]. However, since the proof is short and for the sake of completeness, we reproduce the proof.

Theorem 3. Let $A, B \in M_{n}$. Then $A$ and $B$ are unitarily congruent if and only if $\rho_{A^{*}}(A)=\rho_{A^{*}}(B)$ and $\rho_{B^{*}}(A)=\rho_{B^{*}}(B)$.

Proof. $(\Rightarrow)$ Since $\rho_{C}(A)$ is invariant under unitary congruence of $A$, the result follows. $(\Leftarrow)$ Let $\|\cdot\|$ denote the norm on $M_{n}$ induced by the inner product $\langle A, B\rangle=$ $\operatorname{tr}\left(A B^{*}\right)$ on $M_{n}$.

For any $A, C \in M_{n}$, by Cauchy-Schwarz inequality,

$$
\begin{aligned}
\rho_{C}(A) & =\max \left\{\left|<U^{t} C U, A^{*}>\right|: U \in \mathcal{U}_{n}\right\} \\
& =\max \left\{\left|<A, U^{*} C^{*} U^{*^{*}}>\right|: U \in \mathcal{U}_{n}\right\} \\
& \leq\|A\| \cdot\left\|C^{*}\right\|,
\end{aligned}
$$

with the equality holds if and only if there exists $\alpha \in \mathcal{C}$ and $U \in \mathcal{U}_{n}$ such that $A=$ $\alpha U C^{*} U^{t}$. By the above inequality and given assumption, we have

$$
\|A\|^{2}=\rho_{A^{*}}(A)=\rho_{A^{*}}(B) \leq\|A\| \cdot\|B\|
$$

and

$$
\|B\|^{2}=\rho_{B} \cdot(B)=\rho_{B} \cdot(A) \leq\|A\|\|B\| .
$$

So $\|A\|=\|B\|$ and hence $\rho_{B^{*}}(A)=\|A\| \cdot\|B\|$. Since the equality holds, $A=\alpha U B U^{t}$ for some unitary $U$ and complex number $\alpha$. As $\|A\|=\|B\|$, we can assume $|\alpha|=1$. Then $A=\left(\alpha^{1 / 2} U\right) B\left(\alpha^{1 / 2} U\right)^{t}$ and $\alpha^{1 / 2} U \in \mathcal{U}_{n}$.

\section{Acknowledgements.}

Through private communication, the author learnt that Li and Tsing [4] had also obtained the result of Theorem 1 for the cases $C \in \mathcal{S}_{n}$ or $C \in \mathcal{K}_{n}$ when considering another problem. The author also wishes to thank the referee for his valuable comments on the paper. 


\section{References}

[1] M.D. Choi, C. Laurie, H. Radjavi and P. Rosenthal, On the congruence numerical range and related functions of matrices, Linear and Multilinear Algebra 22 (1987), 1-5.

[2] M. Goldberg and E.G. Straus, Norm properties of C-numerical radii, Linear Algebra and Appl. 24(1979), 113-131.

[3] Y. Hong and R.A. Horn, A characterization of unitary congruence, (to appear in Linear and Mulitinear Algebra).

[4] C.K. Li and N. K. Tsing, G-invariant hermitian forms and G-invariant elliptical norms (to appear).

[5] C.K. Li and N.K. Tsing, Norms that are invariant under unitary similarities and the C-numerical radii (to appear in Linear and Multilinear Algebra).

[6] M. Marcus and M. Sandy, Three elementary proofs of the Goldberg-Straus theorem on numerical radii, Linear and Multilinear Algebra 11 (1982), 243-252.

[7] T. Takagi, On an algebraic problem related to an analytic theorem of Caretheodory and Fejer and on an allied theorem of Landau, Japan J. Math. 1(1925),83-93.

[8] B.S. Tam, A simple proof of the Goldberg-Straus theorem on numerical radii, Glasgow Math. J. 28(1986), 139-141.

[9] T.Y. Tam, Note on a paper of Thompson: The congruence numerical range, Linear and Multilinear Algebra 17 (1985), 107-115.

[10] R.C. Thompson, The congruence numerical range, Linear and Multilinear Algebra 8(1980), 197-206.

[11] D.C. Youla, A normal form for a matrix under the unitary group, Canad. J. Math. 13(1961), 694-704.

Department of Mathematics, University of Hong Kong. 